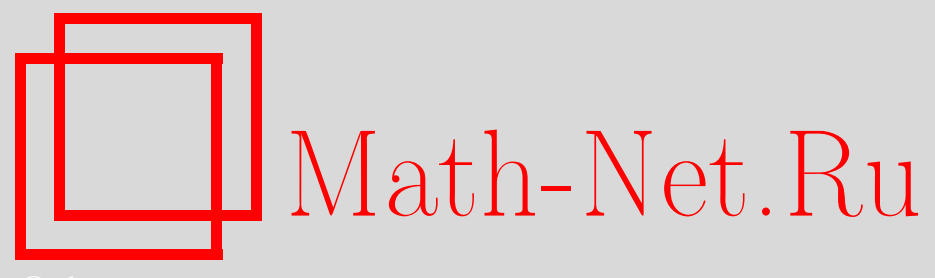

А. В. Тарасов, Универсальные алгебры, порождаемые множествами выполняющих векторов биюнктивных и $r$-юнктивных булевых функций, Матем. вопр. криптогр., 2011, том 2, выпуск $3,75-98$

DOI: https://doi.org/10.4213/mvk37

Использование Общероссийского математического портала Math-Net.Ru подразумевает, что вы прочитали и согласны с пользовательским соглашением http://www.mathnet.ru/rus/agreement

Параметры загрузки:

IP : 34.229 .45 .116

26 апреля 2023 г., 15:58:34 
УДК: 519.571

\title{
Универсальные алгебры, порождаемые множествами выполняющих векторов биюнктивных и $r$-юнктивных булевых функций
}

\author{
A. B. Тарасов \\ Московский государственный технический университет радиотехники, \\ электроники и автоматики, Москва
}

Получено 10.V.2011

В работе введено понятие универсальной алгебры $\Omega_{n}^{r}=\left(V_{n}, v_{r}\right)$, где $V_{n}-$ множество двоичных $n$-мерных векторов, а $v_{r}$ - покоординатно определяемая $(r+1)$-местная операция. Подалгебры этой алгебры образованы множествами выполняющих векторов $r$-юнктивных функций, т. е. функций, представимых в виде $r$-КНФ. В работе описаны эндоморфизмы подалгебр алгебры $\Omega_{n}^{r}$ и их эндоморфные образы. Для случая $r=2$ исследован ряд свойств систем образующих самой алгебры и некоторых подалгебр.

Ключевые слова: биюнктивные функции, $r$-юнктивные функции, $r$-КНФ, универсальная алгебра

Universal algebras generated by sets of satisfying vectors of bijunctive and $r$-junctive Boolean functions

\section{A. V. Tarasov}

Moscow State Technical University of Radio Engineering, Electronics and Automatics, Moscow

Abstract. The notion of universal algebra $\Omega_{n}^{r}=\left(V_{n}, v_{r}\right)$ (where $V_{n}$ is the set of binary
$n$-dimensional vectors and $v_{r}: V_{n}^{r+1} \rightarrow V_{n}$ is the coordinate-wise operation) is introduced.
Subalgebras of this algebra are formed by sets of satisfying vectors for $r$-junctive
functions, i.e. functions which may be represented as $r$-CNF. The endomorphisms of
these subalgebras of algebra $\Omega_{n}^{r}$ and their endomorphic images are described. In the case
of $r=2$ several properties of generating systems of the algebra and of some subalgebras
are investigated. Key words: bijunctive functions, $r$-junctive functions, $r$-CNF, universal algebra Citation: Mathematical Aspects of Cryptography, 2011, vol.2, no.3, pp.75-98 (Russian) 


\section{1. Введение}

Задача решения систем булевых уравнений является одной из актуальных проблем теоретической криптографии. Одним из направлений исследований этой проблемы является изучение классов функций, порождающих полиномиально решаемые системы уравнений с ограничениями на выбор функций и без ограничений на выбор неизвестных. Значительное место в изучении таких классов функций занимают работы Т. Шефера [1], С. П. Горшкова [2-4] и др. Некоторые результаты автора настоящей работы отражены в [5].

Полиномиально решаемые системы уравнений с ограничениями на выбор функций и без ограничений на выбор неизвестных порождаются, например, булевыми биюнктивными функциями, т. е. функциями, представимыми в виде 2-КНФ. Известен целый ряд свойств биюнктивных функций, однако многие вопросы, касающиеся структуры класса биюнктивных функций и множеств их выполняющих векторов, остаются открытыми. В настоящей работе рассматриваются некоторые новые свойства класса биюнктивных функций и одного подкласса класса $r$-юнктивных булевых функций специального вида, основанные на изучении их универсальных алгебр.

\section{2. Алгебры $\Omega_{n}^{r}$ и их основные свойства}

Напомним некоторые определения. Введем следующие классы булевых функций (см., например, [2]). Булева функция $f\left(x_{1}, \ldots, x_{n}\right)$ называется:

1) мультиаффинной, если $f \equiv 1$ или существует представление функции $f$ в виде конъюнкции аффинных функций

$$
f\left(x_{1}, \ldots, x_{n}\right) \equiv \bigwedge_{i=1}^{t}\left(a_{i_{1}} x_{1} \oplus \ldots \oplus a_{i_{n}} x_{n} \oplus a_{i_{0}}\right),
$$

где $a_{i_{0}}, \ldots, a_{i_{n}} \in\{0,1\}, i=1, \ldots, t$;

2) биюнктивной, если $f \equiv 1$ или существует представление функции $f$ в виде 2-КНФ

$$
f\left(x_{1}, \ldots, x_{n}\right) \equiv \stackrel{\curlywedge}{i=1}_{\wedge}^{t}\left(x_{s_{i 1}}^{a_{i 1}} \vee x_{s_{i 2}}^{a_{i 2}}\right),
$$

где $s_{i 1}, s_{i 2} \in\{1, \ldots, n\}, a_{i 1}, a_{i 2} \in\{0,1\}, i=1, \ldots, t, x^{1}=x, x^{0}=\bar{x}$;

3) слабо положительной, если $f \equiv 1$ или существует представление $f$ в виде КНФ

$$
f\left(x_{1}, \ldots, x_{n}\right) \equiv \stackrel{\wedge}{t}_{i=1}^{\wedge}\left(x_{s_{i 1}}^{a_{i}} \vee x_{s_{i 2}} \vee \ldots \vee x_{s_{i k_{i}}}\right)
$$


4) слабо отрицательной, если $f \equiv 1$ или существует представление $f$ в виде КНФ

$$
f\left(x_{1}, \ldots, x_{n}\right) \equiv \stackrel{\curlywedge}{i=1}^{\wedge}\left(x_{s_{i 1}}^{a_{i}} \vee \bar{x}_{s_{i 2}} \vee \ldots \vee \bar{x}_{s_{i k_{i}}}\right),
$$

где $a_{i} \in\{0,1\}, i=1, \ldots, t$;

5) булеву функцию $f$ назовем $r$-юнктивной, если $f \equiv 1$ или существует ее представление в виде конъюнктивной нормальной формы (КНФ) вида

$$
f\left(x_{1}, \ldots, x_{n}\right) \equiv \stackrel{\wedge}{i=1}^{t}\left(x_{s_{i 1}}^{a_{i 1}} \vee x_{s_{i 2}}^{a_{i 2}} \vee \ldots \vee x_{s_{i r_{i}}}^{a_{i_{i}}}\right), r_{i} \leq r, i=1, \ldots, t,
$$

где $s_{i 1}, \ldots, s_{i r_{i}} \in\{1, \ldots, n\}, a_{i 1}, \ldots, a_{i r_{i}} \in\{0,1\}, i=1, \ldots, t$.

Множество всех функций классов 1)-5) обозначим соответственно $A$, $B i, W P, W N, J u_{r}$. Формулы (1)-(5) для функций классов $A, B i, W P, W N, J u_{r}$ будем называть приведенныли представлениями. В работах [2-6] показано, что классы $A, B i, W P, W N$ порождают полиномиально решаемые классы систем уравнений без ограничений на выбор неизвестных и с ограничениями на выбор функций, и исследован ряд их свойств.

Через $F_{2}(n)$ обозначим множество всех булевых функций, зависящих от $n$ переменных.

Очевидно, что

$$
B i \subset J u_{3} \subset J u_{4} \subset \ldots
$$

Если для произвольного класса булевых функций $Q$ определить подкласс $Q(n)$ как множество функций из $Q$, зависящих от $n$ переменных, то будут выполняться очевидные включения

$$
B i(n) \subset J u_{3}(n) \subset J u_{4}(n) \subset \ldots \subset J u_{n}(n)=F_{2}(n) .
$$

К перечисленным множествам функций добавим еще класс $M-$ класс монотонных функций.

Для всякой булевой функции $f\left(x_{1}, \ldots, x_{n}\right)$ определим множество

$$
E_{f}=\left\{\alpha=\left(a_{1}, \ldots, a_{n}\right): f\left(a_{1}, \ldots, a_{n}\right)=1\right\},
$$

которое будем называть множеством выполняющих векторов функции $f$.

Пусть $V_{n}$ - множество двоичных $n$-мерных векторов, $0_{n}-$ вектор из $V_{n}$, состоящий из нулей, $1_{n}-$ вектор из $V_{n}$, состоящий из единиц.

Для множества $N \subset V_{n}$ обозначим через $f_{N}$ булеву функцию, множеством выполняющих векторов которой является множество $N$, т. е. $E_{f_{N}}=N$. 
Определим множество $N \oplus \delta=\{\alpha \oplus \delta: \alpha \in N\}$, где $\delta$ - произвольный вектор из множества $V_{n}$. обозначим

Для всяких $i_{1}, \ldots, i_{r}, 1 \leq i_{1}<\ldots<i_{r} \leq n, a_{1}, \ldots, a_{r} \in\{0,1\}$,

$$
N_{i_{1} \ldots i_{r}}^{a_{1} \ldots a_{r}}=\left\{\alpha=\left(c_{1}, \ldots, c_{n}\right) \in N: c_{i_{j}}=a_{j}, j=1, \ldots, r\right\}
$$

и определим подфункииюю $f_{i_{1} \ldots i_{r}}^{a_{1} \ldots a_{r}}$ булевой функции $f\left(x_{1}, \ldots, x_{n}\right)$ как $(n-r)$-местную функцию, получаемую из $f$ фиксацией переменных $x_{i_{1}}, \ldots, x_{i_{r}}$ значениями $a_{1}, \ldots, a_{r}$.

Координаты вектора $\alpha \in V_{n}$ будем обозначать как $\alpha(1), \ldots, \alpha(n)$. Для векторов $\alpha, \beta \in V_{n}$ запись $\alpha \beta, \alpha \vee \beta$ означает покоординатное применение операций дизъюнкции и конъюнкции.

Введем еще одно обозначение. Пусть $r \geq 2$. Определим $(r+1)$-арную операцию $v_{r}: V_{n}^{r+1} \rightarrow V_{n}$, определенную покоординатно по правилу

$$
v_{r}\left(\alpha_{1}, \alpha_{2}, \ldots, \alpha_{r+1}\right)=\underset{1 \leq i<j \leq r+1}{\vee} \alpha_{i} \alpha_{j},
$$

т. е. если $v_{r}\left(\alpha_{1}, \alpha_{2}, \ldots, \alpha_{r+1}\right)=\delta$, то выполнены равенства

$$
\delta(k)=\underset{1 \leq i<j \leq r+1}{\bigvee} \alpha_{i}(k) \alpha_{j}(k), k=1, \ldots, n .
$$

Заметим, что $k$-я $(k=1, \ldots, n)$ координата вектора $v_{r}\left(\alpha_{1}, \alpha_{2}, \ldots, \alpha_{r+1}\right)$ равна единице тогда и только тогда, когда в векторах $\alpha_{1}, \alpha_{2}, \ldots, \alpha_{r+1}$ по этой координате содержится не менее двух единиц.

Для любого $r \geq 2$ будем рассматривать универсальную алгебру $\Omega_{n}^{r}=$ $=\left(V_{n}, v_{r}\right)$. При этом операцию $v_{2}$ будем обозначать через $v$, а соответствующую алгебру - через $\Omega_{n}$, т. е. $\Omega_{n}=\left(V_{n}, v\right)$.

Для $N \subset V_{n}$ положим $\langle N\rangle^{(1)}=N \cup\left\{v_{r}\left(\alpha_{1}, \ldots, \alpha_{r+1}\right): \alpha_{1}, \ldots, \alpha_{r+1} \in N\right\}$, $\langle N\rangle^{(i+1)}=\left\langle\langle N\rangle^{(i)}\right\rangle^{(1)}, i=1,2, \ldots$ Через $\langle N\rangle$ обозначим подалгебру алгебры $\Omega_{n}^{r}$, порожденную множеством $N$. Очевидно, $\langle N\rangle=\langle N\rangle^{(k)}$ для некоторого $k>0$. Если $\langle N\rangle=N$, то множество $N$ будем называть $v_{r}$-замкнутыл, а если $\langle N\rangle=V_{n}$, то $-v_{r}$-полнылм.

При $n=1$ операция $v_{r}$ является $(r+1)$-местной булевой функцией. 
Утверждение 1. При $n=1$ многочлен Жегалкина функции $v_{r}$ имеет вид

$$
v_{r}\left(x_{1}, \ldots, x_{r+1}\right)=\underset{\substack{k+1 \\ k=2 \\ k \text { четно }}}{\stackrel{r+1}{1}} \bigoplus_{\substack{1 \\ 1} \ldots i_{k} \leq r+1} x_{i_{1}} \ldots x_{i_{k}} .
$$

Доказательство. Пусть $n=1$. Определим функцию $w_{r}=v_{r} \oplus 1$. Согласно определению данная функция равна единице только на вектоpax веса не более 1. Найдем коэффициенты ее многочлена Жегалкина $a_{0}, a_{1}, \ldots, a_{r+1}, a_{12}, \ldots, a_{12 \ldots r+1}$. Ввиду того что функция симметрическая, для ее коэффициентов выполняются равенства $a_{12 \ldots k}=a_{i_{1} \ldots i_{k}}$ для всех $k \in\{1,2, \ldots, r+1\}, 1 \leq i_{1}<\ldots<i_{k} \leq r+1$.

Поэтому положим $b_{k}=a_{1 \ldots k}, 1 \leq k \leq r+1, b_{0}=a_{0}$, и применим метод неопределенных коэффициентов. Имеем

$$
\begin{gathered}
b_{0}=a_{0}=w_{r}(0, \ldots, 0)=1, \\
b_{1}=w_{r}(1,0, \ldots, 0) \oplus b_{0}=0, \\
b_{2}=w_{r}(1,1, \ldots, 0) \oplus b_{0} \oplus b_{1} \oplus b_{1}=1 .
\end{gathered}
$$

Очевидно, что во введенных обозначениях при $k \geq 2$ верны равенства

$$
b_{k}=\bigoplus_{i=0}^{k-1}\left(\left(C_{k}^{i} \bmod 2\right) \& b_{i}\right) .
$$

Покажем, что $b_{k}=1$ при четном $k$ и $b_{k}=0$ при нечетном $k$. Доказательство проведем индукцией по $k$. Базис индукции - значения $b_{0}, b_{1}, b_{2}$, подсчитанные выше. Пусть утверждение верно для $b_{0}, \ldots, b_{k-1}$ и $k$ четно. Тогда

$$
\begin{aligned}
& b_{k}=\underset{\substack{i=0 \\
i \text { четно }}}{\bigoplus}\left(C_{k}^{i} \bmod 2\right)=\left(\underset{\substack{i=0 \\
i \text { четно }}}{\sum_{k}-1} C_{k}^{i}\right) \bmod 2= \\
& =\left(\sum_{\substack{i=0 \\
i \text { четно }}}^{k} C_{k}^{i}-C_{k}^{k}\right) \bmod 2=\left(2^{k-1}-1\right) \bmod 2=1 .
\end{aligned}
$$

Если же $k$ нечетно, то

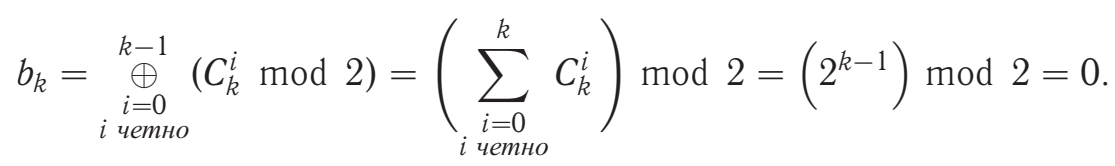

Из этих рассуждений о виде последовательности $b_{k}, k=1,2, \ldots, r+1$, легко следует искомый результат. 
В следующем утверждении доказывается еще одно важное свойство операции $v_{r}$.

Утверждение 2. Пусть $\alpha_{1}, \ldots, \alpha_{r+1}, \delta \in V_{n} u \quad \delta=v_{r}\left(\alpha_{1}, \ldots, \alpha_{r+1}\right)$. Тогда для любых $i_{1}, \ldots, i_{r} \in\{1, \ldots, n\}$ среди векторов $\alpha_{1}, \ldots, \alpha_{r+1}$ найдется такой вектор $\alpha_{m} \in\left\{\alpha_{1}, \ldots, \alpha_{r+1}\right\}$, что $\delta\left(i_{1}\right)=\alpha_{m}\left(i_{1}\right), \ldots, \delta\left(i_{r}\right)=\alpha_{m}\left(i_{r}\right)$.

Доказательство. Предположим противное. Без ограничения общности будем считать, что $i_{1}=1, \ldots, i_{r}=r$ и $\delta(1)=\ldots=\delta(r)=0$. В соответствии со сделанным предположением среди векторов $\alpha_{1}, \ldots, \alpha_{r+1}$ не найдется вектора, первые $r$ координат которого равны 0. Это означает, что у каждого из векторов $\alpha_{1}, \ldots, \alpha_{r+1}$ содержится по крайней мере по одной единице на местах $1, \ldots, r$. Следовательно, суммарное число единиц среди двоичных чисел $\alpha_{1}(1), \ldots, \alpha_{1}(r), \ldots, \alpha_{r+1}(r)$ не меньше $r+1$. С другой стороны, поскольку $\delta=v_{r}\left(\alpha_{1}, \ldots, \alpha_{r+1}\right)$ и $\delta(1)=\ldots=\delta(r)=0$, то в каждом из векторов $\beta_{1}=\left(\alpha_{1}(1), \ldots, \alpha_{r+1}(1)\right), \ldots, \beta_{r}=\left(\alpha_{1}(r), \ldots, \alpha_{r+1}(r)\right)$ содержится не более одной единицы, что противоречит сделанному выше выводу о суммарном числе единиц. Полученное противоречие доказывает утверждение.

В работе Шефера [1] доказан критерий биюнктивности, который во введенных обозначениях можно сформулировать следующим образом.

Теорема 1. Булева функиия $f\left(x_{1}, \ldots, x_{n}\right)$ биюнктивна тогда и только тогда, когда множество $E_{f}$ является v-замкнутым, т.е. является подалгеброй алгебры $\Omega_{n}$.

Следующая теорема реализует идею А. С. Артемьева о том, что критерий биюнктивности Шефера можно обобщить на более широкий класс функций. Прежде чем перейти к ее формулировке и доказательству, напомним [4], что класс функций $W P \cap W N$ является подклассом класса $B i$ и состоит из функций $f\left(x_{1}, \ldots, x_{n}\right)$, представимых в виде 2 -КНФ

$$
f\left(x_{1}, \ldots, x_{n}\right) \equiv \bigwedge_{i=1}^{t}\left(\bar{x}_{s_{i 1}} \vee x_{s_{i 2}}\right) .
$$

Класс $M \cap J u_{r}$ определим как класс монотонных $r$-юнктивных функций. Несложно показать, что такие функции представимы КНФ вида

$$
f\left(x_{1}, \ldots, x_{n}\right) \equiv \underset{i=1}{\wedge}\left(x_{s_{i 1}} \vee x_{s_{i 2}} \vee \ldots \vee x_{s_{i l_{i}}}\right), \quad l_{i} \leq r, \quad i=1, \ldots, t .
$$

Имплицентой булевой функции $f$ назовем такую элементарную дизъюнкцию $D$, что $f \leq D$ на всех наборах значений переменных. Имплицента называется 
простой, если никакая ее собственная часть не является имплицентой функции $f$. Таким образом, понятие имплиценты является двойственным к широко известному понятию импликанты. Поэтому общеизвестные утверждения, касающиеся свойств импликант, можно двойственным образом сформулировать и для имплицент.

Теорема 2. Пусть $f\left(x_{1}, \ldots, x_{n}\right)-$ булева функиия $и r \geq 3$. Множество $E_{f}$ является $v_{r}$-замкнутым тогда и только тогда, когда существует представление функиии $f$ в виде $f\left(x_{1}, \ldots, x_{n}\right)=g h$, где $g \in W P \cap W N$, $h \in M \cap J u_{r}$.

Доказательство. Достаточность. Пусть функция $f\left(x_{1}, \ldots, x_{n}\right)$ имеет представление указанного вида и $\bigwedge_{i=1}^{\mu} D_{i}-$ соответствующая КНФ. Пусть теперь $\left\{\alpha_{1}, \ldots, \alpha_{r+1}\right\}$ - произвольный набор из $r+1$ выполняющих векторов функции $f\left(x_{1}, \ldots, x_{n}\right)$ (не обязательно различных). Очевидно, что произвольный вектор из этого набора является выполняющим для каждой элементарной дизъюнкции $D_{i}, i \in 1, \ldots, \mu$. Покажем, что при этом вектор $\delta=$ $=v_{r}\left(\alpha_{1}, \ldots, \alpha_{r+1}\right)$ также является выполняющим для дизъюнкции $D_{i}, i \in$ $\in 1, \ldots, \mu$, из чего и будет следовать, что вектор $\delta$ является также выполняющим и для функции $f\left(x_{1}, \ldots, x_{n}\right)$. С точностью до перестановки переменных элементарные дизьюнкции $D_{i}$ будут иметь следующий вид:

либо $D=x_{1} \vee \ldots \vee x_{l}$, где $l \leq r$,

либо $D=\bar{x}_{1} \vee x_{2}$.

Рассмотрим первый случай. Из соотношения $\alpha_{1}, \ldots, \alpha_{r+1} \in E_{f}$ следует, что $\alpha_{1}, \ldots, \alpha_{r+1} \in E_{D}$. Значит, каждый из этих векторов содержит по крайней мере по одной единице на местах с 1-го по $l$-е. Следовательно, найдется такая координата $i: 1 \leq i \leq l$, что по крайней мере два из указанных векторов имеют 1 на $i$-м месте. Тогда если $\delta=v_{r}\left(\alpha_{1}, \ldots, \alpha_{r}+1\right)$, то $\delta(i)=1$ и $\delta \in E_{D}$.

Пусть теперь $D=\bar{x}_{1} \vee x_{2}$. Если $\alpha_{1}, \ldots, \alpha_{r+1} \in E_{f}$, то в первых двух координатах этих векторов не может встречаться комбинация $(1,0)$. Пусть $\delta=v_{r}\left(\alpha_{1}, \ldots, \alpha_{r+1}\right)$. Если в первых координатах векторов $\alpha_{1}, \ldots, \alpha_{r+1}$ не более одной единицы, то $\delta(1)=0$ и тогда $\delta \in E_{f}$. Пусть $\delta(1)=1$. Тогда найдутся такие два вектора $\alpha_{i}, \alpha_{j} \in\left\{\alpha_{1}, \ldots, \alpha_{r+1}\right\}$, что $\alpha_{i}(1)=\alpha_{j}(1)=1$. Поскольку эти векторы - выполняющие для $D$, для них выполняются равенства $\alpha_{i}(2)=\alpha_{j}(2)=1$, откуда $\delta(2)=1$ и $\delta \in E_{D}$. Указанные рассуждения верны для любой простой имплиценты $f$. Достаточность доказана.

Необходимость. Предположим, что для булевой функции $f\left(x_{1}, \ldots, x_{n}\right)$ множество $E_{f}$ является $v_{r}$-замкнутым. Поскольку $r \geq 3$, для любых $\alpha_{1}, \alpha_{2} \in E_{f}$ 
верно включение

$$
v_{r}\left(\alpha_{1}, \alpha_{1}, \alpha_{2}, \alpha_{2}, \ldots, \alpha_{2}\right)=\alpha_{1} \vee \alpha_{2} \in E_{f} .
$$

В работе [3] доказан критерий слабой положительности, в соответствии с которым функция является слабо положительной тогда и только тогда, когда множество ее выполняющих векторов замкнуто относительно операции покоординатной дизъюнкции. Следовательно, $f \in W P$.

Рассмотрим соответствующее приведенное представление функции $f$ вида (3). Имплиценты функции $f$ имеют вид либо $D=x_{1} \vee \ldots \vee x_{q}$, либо $D=\bar{x}_{1} \vee x_{2} \vee \ldots \vee x_{q}$.

Рассмотрим первый случай. Будем считать, что имплицента $D$ простая, т. е. никакая ее часть не является имплицентой $f$. Это означает, что для всякого $i, 1 \leq i \leq q$, функция $f_{1 \ldots i}^{0 \ldots . . .0}$ не равна тождественно нулю. Построим $q$ выполняющих векторов

$$
\begin{aligned}
& \alpha_{1}=\left(1,0, \ldots, 0,0, \alpha_{1}(q+1), \ldots, \alpha_{1}(n)\right), \\
& \alpha_{2}=\left(0,1, \ldots, 0,0, \alpha_{2}(q+1), \ldots, \alpha_{2}(n)\right), \\
& \left.\ldots \quad \ldots \quad \ldots \quad \ldots \quad \ldots \quad \ldots, \ldots, \ldots, \alpha_{q}(n)\right) . \\
& \alpha_{q}=\left(0,0, \ldots, 0,1, \alpha_{q}(q+1), \ldots,\right.
\end{aligned}
$$

Легко видеть, что при $q \geq r+1$ применение операции $v_{r}$ к любым $r+1$ векторам из данной совокупности даст вектор

$$
\delta=(0,0, \ldots, 0,0, \delta(q+1), \ldots, \delta(n)) \notin E_{D} .
$$

Такой вектор не лежит в $E_{f}$. Получили противоречие.

Рассмотрим случай имплиценты $D=\bar{x}_{1} \vee x_{2} \vee \ldots \vee x_{q}$. Покажем, что в этом случае $q \leq 2$. Аналогично вышесказанному построим $q$ выполняющих векторов

$$
\begin{aligned}
\alpha_{1}= & \left(0,0, \ldots, 0,0, \alpha_{1}(q+1), \ldots, \alpha_{1}(n)\right), \\
\alpha_{2}= & \left(1,1, \ldots, 0,0, \alpha_{2}(q+1), \ldots, \alpha_{2}(n)\right), \\
\ldots & \ldots \quad \ldots \quad \ldots \quad \ldots \quad \ldots \quad \ldots \quad \ldots \\
\alpha_{q}= & \left(1,0, \ldots, 0,1, \alpha_{q}(q+1), \ldots, \alpha_{q}(n)\right) .
\end{aligned}
$$

При $q \geq 3$ получаем, что

$$
\delta=v_{r}\left(\alpha_{1}, \ldots, \alpha_{1}, \alpha_{2}, \alpha_{3}\right)=(1,0, \ldots, 0, \delta(q+1), \ldots, \delta(n)) \notin E_{f} .
$$

Таким образом, необходимость доказана.

Доказанная теорема показывает, что подалгебры алгебры $\Omega_{n}^{r}$ при $r \geq 3$ являются множествами выполняющих векторов для функций специального вида из подкласса класса $J u_{r}$. Указанный класс функций обозначим через $J u_{r}^{1}$. 
Определение 1. Множество $N \subset V_{n}$ называется комбинаторно полным порядка $r$, если для любых таких $i_{1}, \ldots, i_{r}$, что $1 \leq i_{1}<\ldots<i_{r} \leq n$, и $a_{1}, \ldots, a_{r} \in\{0,1\}$, в множестве $N$ найдется такой вектор $\alpha=\left(c_{1}, \ldots, c_{n}\right)$, что $c_{i_{s}}=a_{s}, s=1,2, \ldots, r$.

Напомним, что наилучшим верхним биюнктивным аналогом булевой функции $f$ называется биюнктивная функция $g$, удовлетворяющая условиям: $g \geq f$ и если для некоторой биюнктивной функции $h$ выполнено неравенство $h \geq f$, то верно неравенство $h \geq g$ ( [3]).

Теорема 3. Пусть $N \subset V_{n}$. Для наилучшего верхнего биюнктивного аналога $g$ функции $f=f_{N}$ верно равенство $E_{g}=\langle N\rangle$. Множество $N$ v-полно тогда и только тогда, когда оно комбинаторно полно порядка 2.

Доказательство. По теореме 1 функция $g$, определяемая равенством $E_{g}=\langle N\rangle$, является биюнктивной. Кроме того, очевидно, что $g \geq f$. Далее, для всякой такой биюнктивной функции $h\left(x_{1}, \ldots, x_{n}\right)$, что $h \geq f$, верно включение $N=E_{f} \subset E_{h}$. Кроме того, опять же по теореме $1,\left\langle E_{h}\right\rangle=E_{h}$. Поэтому $E_{g}=\langle N\rangle \subset\left\langle E_{h}\right\rangle=E_{h}$ и $h \geq g$. Первая часть теоремы доказана.

Перейдем к доказательству второй части теоремы. Сначала покажем, что если $g-$ наилучший верхний биюнктивный аналог $f$, то верно равенство

$$
g\left(x_{1}, \ldots, x_{n}\right)=\bigwedge_{i=1}^{t}\left(x_{i_{1}}^{a_{1} \oplus 1} \vee x_{i_{2}}^{a_{2} \oplus 1}\right),
$$

где конъюнкция берется по всем таким наборам $\left(i_{1}, i_{2}, a_{1}, a_{2}\right)$, что $i_{1}, i_{2} \in$ $\in\{1, \ldots, n\}, a_{1}, a_{2} \in\{0,1\}$ и $\left(f_{M}\right)_{i_{1} i_{2}}^{a_{1} a_{2}} \equiv 0$.

Докажем включение $\langle N\rangle \subset E_{g}$. В самом деле, если для некоторых $i_{1}, i_{2}$, $1 \leq i_{1}<i_{2} \leq n, a_{1}, a_{2} \in\{0,1\}$, выполнено условие $f_{i_{1} i_{2}}^{a_{1} a_{2}} \equiv 0$, то у любого вектора $\alpha \in M$ координаты $\left(\alpha\left(i_{1}\right), \alpha\left(i_{2}\right)\right)$ не могут принимать значения $\left(a_{1}, a_{2}\right)$. Соответственно, для любого набора $\alpha_{1}, \alpha_{2}, \alpha_{3} \in N$ их комбинация $\delta=v\left(\alpha_{1}, \alpha_{2}, \alpha_{3}\right)$ обладает таким же свойством по утверждению 2. Данным свойством обладают также множества $\langle N\rangle^{(1)},\langle N\rangle^{(2)}, \ldots,\langle N\rangle$. Максимальное по включению множество с указанным свойством - множество $E_{\phi}$, где $\phi=x_{i_{1}}^{a_{1} \oplus 1} \vee x_{i_{2}}^{a_{2} \oplus 1}$. Поэтому $\langle N\rangle \subset E_{\phi}$. Проведя подобные рассуждения для всех таких $i_{1}, i_{2}, 1 \leq i_{1}<i_{2} \leq n, a_{1}, a_{2} \in\{0,1\}$, что $f_{i_{1} i_{2}}^{a_{1} a_{2}} \equiv 0$, получим включение $\langle N\rangle \subset E_{g}$.

Докажем обратное включение $E_{g} \subset\langle N\rangle$. Предположим противное, т. е. что существует вектор $\alpha \in E_{g} \backslash\langle N\rangle$. По критерию биюнктивности функция $f_{\langle N\rangle}$ биюнктивна, следовательно, $f \leq f_{\langle N\rangle} \leq g$. Получили противоречие. Равенство (6) доказано. 
Докажем вторую часть теоремы. Пусть множество $N$ не является комбинаторно полным порядка 2 , т. е. $N_{i_{1} i_{2}}^{a_{1} a_{2}}=\emptyset$ для некоторых $i_{1}, i_{2}, 1 \leq i_{1}<$ $<i_{2} \leq n, a_{1}, a_{2} \in\{0,1\}$. Рассмотрим функцию $f_{N}$. Очевидно, что $f_{N} \leq \phi=$ $=x_{i_{1}}^{a_{1} \oplus 1} \vee x_{i_{2}}^{a_{2} \oplus 1}$, т. е. функция $f_{N}$ имеет нетривиальный верхний биюнктивный аналог. Тогда, в соответствии с первой частью теоремы, $\langle N\rangle \subset E_{\phi} \neq V_{n}$.

Пусть теперь множество $N$ комбинаторно полно порядка 2. Покажем, что оно v-полно. Так как $N \subset\langle N\rangle$, то функция $g=f_{\langle N\rangle}$ биюнктивна и $f_{\langle N\rangle} \geq f_{N}$. Если $\langle N\rangle \neq V_{n}$, то функция $f_{N}$ имеет нетривиальный верхний биюнктивный аналог, а значит, $N_{i_{1} i_{2}}^{a_{1} a_{2}}=\emptyset$ для некоторых $i_{1}, i_{2}, 1 \leq i_{1}<i_{2} \leq n$, $a_{1}, a_{2} \in\{0,1\}$, что противоречит заявленному свойству множества $N$.

Теорема 3 показывает, что задача изучения биюнктивных функций сводится к задаче изучения подалгебр алгебры $\Omega_{n}$.

Напомним (см. [7, стр. 101]), что применительно к рассматриваемому случаю элементарной транслящией $(r+1)$-местной операции $v_{r}$ называется унарная операция, полученная из исходной фиксацией любых $r$ переменных произвольными значениями.

Теорема 4. При $r \geq 2$ множество элементарных трансляций операиии $v_{r}$ совпадает с множеством

$$
\mathrm{T}_{n}=\left\{\tau_{\alpha \beta}(x): V_{n} \rightarrow V_{n}, \tau_{\alpha \beta}(x)=\alpha x \oplus \beta, \alpha, \beta \in V_{n}, \alpha \beta=0_{n}\right\} .
$$

Доказательство. Рассмотрим произвольную элементарную трансляцию

$$
v_{r}\left(\alpha_{1}, \ldots, \alpha_{r}, x\right): V_{n} \rightarrow V_{n},
$$

где $\alpha_{1}, \ldots, \alpha_{r}-$ фиксированные векторы. В соответствии с определением операции $v_{r}$ верны равенства

$$
\begin{aligned}
& v_{r}\left(\alpha_{1}, \ldots, \alpha_{r}, x\right)=\alpha_{1} \alpha_{2} \vee \ldots \vee \alpha_{1} \alpha_{r} \vee \alpha_{1} x \vee \ldots \vee \alpha_{r} x= \\
& =\left(\alpha_{1} \vee \ldots \vee \alpha_{r}\right) x \vee \alpha_{1} \alpha_{2} \vee \ldots \vee \alpha_{1} \alpha_{r} \vee \ldots \vee \alpha_{r-1} \alpha_{r}= \\
& =\left(\alpha_{1} \vee \ldots \vee \alpha_{r}\right) x \vee v_{r-1}\left(\alpha_{1}, \ldots, \alpha_{r}\right) .
\end{aligned}
$$

Положим $\gamma=\alpha_{1} \vee \ldots \vee \alpha_{r}, \beta=v_{r-1}\left(\alpha_{1}, \ldots, \alpha_{r}\right)$. Тогда

$$
v_{r}\left(\alpha_{1}, \ldots, \alpha_{r}, x\right)=\gamma x \vee \beta .
$$


Теперь построим вектор $\alpha=\gamma\left(\beta \oplus 1_{n}\right)$. Ясно, что $\alpha \beta=0_{n}$. При этом выполняется цепочка равенств

$$
\alpha x \oplus \beta=\gamma(\beta \oplus 1) x \oplus \beta=(\gamma x) \oplus(\gamma x) \beta \oplus \beta=\gamma x \vee \beta .
$$

Таким образом, показано, что $v_{r}\left(\alpha_{1}, \ldots, \alpha_{r}, x\right) \in \mathrm{T}_{n}$.

Теперь докажем, что любое отображение из $\mathrm{T}_{n}$ является элементарной трансляцией. Пусть $\tau_{\alpha \beta} \in \mathrm{T}_{n}, \alpha, \beta \in V_{n}, \alpha \beta=0_{n}$. Построим такие векторы $\alpha_{1}, \ldots, \alpha_{r}$, что $v_{r}\left(\alpha_{1}, \ldots, \alpha_{r}, x\right)=\alpha x \oplus \beta$.

В случае, когда $\alpha=0_{n}$, положим $\alpha_{1}=\beta, \ldots, \alpha_{r}=\beta$. Тогда очевидно, что $\tau_{\alpha \beta}(x) \equiv \beta \equiv v_{r}(\beta, \ldots, \beta, x)$.

Пусть $\alpha \neq 0_{n}$. Сначала рассмотрим случай $r=2$. Положим $\alpha_{1}=$ $=\beta, \alpha_{2}=\alpha \vee \beta$. Тогда

$$
\begin{aligned}
& v\left(\alpha_{1}, \alpha_{2}, x\right)=v(\alpha \vee \beta, \beta, x)=(\alpha \vee \beta) x \oplus \beta x \oplus(\alpha \vee \beta) \beta= \\
& =(\alpha \beta \oplus \alpha \oplus \beta) x \oplus \beta x \oplus \beta=(\alpha \oplus \beta) x \oplus \beta x \oplus \beta=\alpha x \oplus \beta .
\end{aligned}
$$

Положив теперь $\alpha_{3}=\ldots=\alpha_{r}=0$, получим, что $v_{r}\left(\alpha_{1}, \ldots, \alpha_{r}, x\right)=\alpha x \oplus \beta$.

Для числа элементарных трансляций верны следующие утверждения.

Утверждение 3. Для числа элементов множества $\mathrm{T}_{n}$ верно равенство $\left|\mathrm{T}_{n}\right|=3^{n}$.

Доказательство. Для подсчета числа элементарных трансляций достаточно посчитать количество таких пар $(\alpha, \beta)$, что $\alpha, \beta \in V_{n}, \alpha \beta=0_{n}$. Так как для разных пар $(\alpha, \beta)$ трансляции, очевидно, различны, то

$$
\left|\mathrm{T}_{n}\right|=\sum_{k=0}^{n} C_{n}^{k} 2^{n-k}=\sum_{k=0}^{n} C_{n}^{k} 2^{k}=3^{n} .
$$

Утверждение 4. Множество преобразований $\mathrm{T}_{n}$ образует относительно операции суперпозиции некоммутативную полугруппу с единицей, каждый элемент которой является идемпотентом.

Доказательство. Ассоциативность следует из ассоциативности операции композиции отображений. Докажем замкнутость:

$$
\tau_{\alpha_{1} \beta_{1}}\left(\tau_{\alpha_{2} \beta_{2}}(x)\right)=\alpha_{1}\left(\alpha_{2} x \oplus \beta_{2}\right) \oplus \beta_{1}=\alpha_{1} \alpha_{2} x \oplus\left(\alpha_{1} \beta_{2} \oplus \beta_{1}\right)=\tau_{\alpha_{1} \alpha_{2}, \alpha_{1} \beta_{2} \oplus \beta_{1}} .
$$

Единицей полугруппы является тождественное отображение $\tau_{1_{n} 0_{n}}$. 
Идемпотентность следует из равенства

$$
\tau_{\alpha \beta}\left(\tau_{\alpha \beta}(x)\right)=\alpha(\alpha x \oplus \beta) \oplus \beta=\alpha x \oplus \alpha \beta \oplus \beta=\alpha x \oplus \beta .
$$

\section{3. Эндоморфизмы алгебр $\Omega_{n}^{r}$}

Определим универсальную алгебру $\Psi_{n}=\left(V_{n}, \mathrm{~T}_{n}\right)$.

ЗАМЕЧАНИЕ 1. Согласно [7, с. 102] множество конгруэнций алгебры $\Psi_{n}$ совпадает с множеством конгруэнций алгебры $\Omega_{n}^{r}$.

Пусть $\phi: V_{n} \rightarrow V_{n}$ - преобразование множества $V_{n}$ с координатными функциями $\phi_{1}, \ldots, \phi_{n}$. Определим бинарные отношения $\varepsilon_{\phi_{1}}, \ldots, \varepsilon_{\phi_{n}}$ на $V_{n}$ по принципу: если $x, y \in V_{n}$, то $x \varepsilon_{\phi_{i}} y$ тогда и только тогда, когда $\phi_{i}(x)=$ $=\phi_{i}(y), i=1,2, \ldots, n$. Также определим бинарное отношение $\varepsilon_{\phi}: x \varepsilon_{\phi} y$ тогда и только тогда, когда $\phi(x)=\phi(y)$. Фактически $\varepsilon_{\phi}=\varepsilon_{\phi_{1}} \cap \ldots \cap \varepsilon_{\phi_{n}}$. Если $\phi-$ эндоморфизм алгебры $\Omega_{n}^{r}$, то отношения $\varepsilon_{\phi}, \varepsilon_{\phi_{1}}, \ldots, \varepsilon_{\phi_{n}}$ будут конгруэнциями на $\Omega_{n}^{r}$. Следовательно, по замечанию 1 , они будут конгруэнциями алгебры $\Psi_{n}$.

Лемма 1. Пусть $r \geq 2, \phi=\left(\phi_{1}, \ldots, \phi_{n}\right): V_{n} \rightarrow V_{n}-$ эндоморфизм алгебры $\Omega_{n}^{r}$ и существуют такие различные векторы $y, z, \in V_{n}$, что $\phi(y)=$ $=\phi(z)$. Тогда если для некоторого $i \in\{1,2, \ldots, n\}$ выполнено условие $y(i) \neq$ $\neq z(i)$, то функиии $\phi_{1}, \ldots, \phi_{n}$ не зависят существенно от переменной $x_{i}$.

Доказательство. Без ограничения общности будем считать, что $i=1$ и $y=(0, y(2), \ldots, y(n)), z=(1, z(2), \ldots, z(n))$. Предположим, что функция $\phi_{j}\left(x_{1}, \ldots, x_{n}\right), j \in\{1,2, \ldots, n\}$, существенно зависит от переменной $x_{1}$. Тогда найдутся такие соседние векторы $\gamma=(0, \gamma(2), \ldots, \gamma(n)), \gamma^{\prime}=$ $=(1, \gamma(2), \ldots, \gamma(n))$, что $\phi_{j}(\gamma) \neq \phi_{j}\left(\gamma^{\prime}\right)$. Так как $\phi-$ гомоморфизм $\Omega_{n}^{r}$, то $\varepsilon_{\phi}-$ конгруэнция $\Omega_{n}^{r}$. Следовательно, $\varepsilon_{\phi_{j}}-$ также конгруэнция $\Omega_{n}^{r}$, а значит, и конгруэнция алгебры $\Psi_{n}$ в силу сделанного выше замечания 1.

Так как $\phi(y)=\phi(z)$, то $y \varepsilon_{\phi} z$, откуда $y \varepsilon_{\phi_{j}} z$. Поэтому для любых $\alpha, \beta \in V_{n}: \alpha \beta=0_{n}$ должно выполняться условие $\tau_{\alpha \beta}(y) \varepsilon_{\phi_{j}} \tau_{\alpha \beta}(z)$, т. е. $\phi_{j}\left(\tau_{\alpha \beta}(y)\right)=\phi_{j}\left(\tau_{\alpha \beta}(z)\right)$. Положим $\alpha=(1,0, \ldots, 0), \beta=\gamma$. Тогда

$$
\tau_{\alpha \beta}(y)=\gamma, \tau_{\alpha \beta}(z)=\gamma^{\prime}
$$


и

$$
\phi_{j}\left(\tau_{\alpha \beta}(y)\right)=\phi_{j}(\gamma) \neq \phi_{j}\left(\tau_{\alpha \beta}(z)\right)=\phi_{j}\left(\gamma^{\prime}\right) .
$$

Полученное противоречие доказывает лемму.

Обозначим полугруппу эндоморфизмов алгебры $\Omega_{n}^{r}$ через $\operatorname{End}\left(\Omega_{n}^{r}\right)$. Через $\Xi_{n}$ обозначим множество отображений $\phi=\left(\phi_{1}, \ldots, \phi_{n}\right): V_{n} \rightarrow V_{n}$, каждая из координатных функций $\phi_{1}, \ldots, \phi_{n}$ которых существенно зависит не более, чем от одной переменной, и положим $\Xi_{n}^{0}-$ множество преобразований $\phi=\left(\phi_{1}, \ldots, \phi_{n}\right) \in \Xi_{n}$, удовлетворяющих условию: если координатная функция $\phi_{i}, i=1,2, \ldots, n$, существенно зависит от одной переменной, то $\phi_{i}\left(0_{n}\right)=0$. В частности, для таких групп преобразований пространства $V_{n}$, как симметрическая группа перестановок координат $S_{n}$ и группа Джевонса $Q_{n}$, верны включения $Q_{n} \subset \Xi_{n}, S_{n} \subset \Xi_{n}^{0}$. Приводимая ниже теорема для случая $r=2$ в некотором смысле обобщает полученный ранее результат автора (см. [8, теорема 2]).

Теорема 5. Пусть $\phi=\left(\phi_{1}, \ldots, \phi_{n}\right): V_{n} \rightarrow V_{n}$. Для того чтобы выполнялось условие $\phi \in \operatorname{End}\left(\Omega_{n}\right)$, необходимо и достаточно, чтобы $\phi \in \Xi_{n}$. Получаемые при таких эндоморфизмах образы алгебры $\Omega_{n}$ будут подалгебрами $\Omega_{n}$, изоморфными алгебрам $\Omega_{0}, \ldots, \Omega_{n-1}, \Omega_{n}$, а их основные множества будут совпадать с множествами выполняющих векторов мультиаффинных биюнктивных функиий.

Доказательство. Сначала докажем первую часть теоремы в прямую сторону. Пусть $\phi \in \operatorname{End}\left(\Omega_{n}\right)$.

1. Сначала рассмотрим случай, когда $\phi\left(V_{n}\right) \neq V_{n}$. В этом случае должны существовать хотя бы два вектора с равными образами при действии преобразования $\phi$. Обозначим эти векторы через $y$ и $z$ и, без ограничения общности, будем считать, что они различны по первой координате: $y=$ $=(0, y(2), \ldots, y(n)), z=(1, z(2), \ldots, z(n))$. По лемме 1 функции $\phi_{1}, \ldots, \phi_{n}$ не зависят существенно от первой переменной. Не ограничивая общности доказательства, рассмотрим функцию $\phi_{1}\left(x_{1}, \ldots, x_{n}\right)$. Предположим, что она существенно зависит хотя бы от двух переменных: $x_{2}$ и $x_{3}$. Тогда найдутся две такие пары соседних векторов вида:

$$
\begin{aligned}
& \gamma=(\gamma(1), 0, \gamma(3), \gamma(4), \ldots, \gamma(n)) \\
& \gamma^{\prime}=(\gamma(1), 1, \gamma(3), \gamma(4), \ldots, \gamma(n)) \\
& \delta=(\delta(1), \delta(2), 0, \delta(4), \ldots, \delta(n)) \\
& \delta^{\prime}=(\delta(1), \delta(2), 1, \delta(4), \ldots, \delta(n))
\end{aligned}
$$


что $\phi_{1}(\gamma) \neq \phi_{1}\left(\gamma^{\prime}\right), \phi_{1}(\delta) \neq \phi_{1}\left(\delta^{\prime}\right)$. Поскольку $x_{1}-$ несущественная переменная $\phi_{1}$, можно считать, что $\gamma(1)=\delta(1)=0$. Построим следующее множество векторов:

$$
\begin{aligned}
& \gamma_{0}=(0,0, \gamma(3), \gamma(4), \ldots, \gamma(n)), \\
& \gamma_{1}=(1,0, \gamma(3), \gamma(4), \ldots, \gamma(n)), \\
& \gamma_{2}=(0,1, \gamma(3), \gamma(4), \ldots, \gamma(n)), \\
& \gamma_{3}=(1,1, \gamma(3), \gamma(4), \ldots, \gamma(n)), \\
& \delta_{0}=(0, \delta(2), 0, \delta(4), \ldots, \delta(n)), \\
& \delta_{1}=(1, \delta(2), 0, \delta(4), \ldots, \delta(n)), \\
& \delta_{2}=(0, \delta(2), 1, \delta(4), \ldots, \delta(n)), \\
& \delta_{3}=(1, \delta(2), 1, \delta(4), \ldots, \delta(n))
\end{aligned}
$$

Для этого набора векторов будут выполняться соотношения

$$
\begin{aligned}
& \phi_{1}\left(\gamma_{0}\right)=\phi_{1}\left(\gamma_{1}\right) \neq \phi_{1}\left(\gamma_{2}\right)=\phi_{1}\left(\gamma_{3}\right), \\
& \phi_{1}\left(\delta_{0}\right)=\phi_{1}\left(\delta_{1}\right) \neq \phi_{1}\left(\delta_{2}\right)=\phi_{1}\left(\delta_{3}\right) .
\end{aligned}
$$

Исходя из множества векторов (7) со свойством (8), построим такие наборы векторов $\left\{\alpha_{1}, \alpha_{2}, \alpha_{3}\right\},\left\{\beta_{1}, \beta_{2}, \beta_{3}\right\}$, что

$$
\alpha_{1} \varepsilon_{\phi_{1}} \beta_{1}, \alpha_{2} \varepsilon_{\phi_{1}} \beta_{2}, \alpha_{3} \varepsilon_{\phi_{1}} \beta_{3},
$$

но

$$
\phi_{1}\left(v\left(\alpha_{1}, \alpha_{2}, \alpha_{3}\right)\right) \neq \phi_{1}\left(v\left(\beta_{1}, \beta_{2}, \beta_{3}\right)\right) .
$$

Если $\gamma(3)=\delta(2)=c, c \in\{0,1\}$, то (7) принимает вид:

$$
\begin{aligned}
& \gamma_{0}=(0,0, c, \gamma(4), \ldots, \gamma(n)), \\
& \gamma_{1}=(1,0, c, \gamma(4), \ldots, \gamma(n)), \\
& \gamma_{2}=(0,1, c, \gamma(4), \ldots, \gamma(n)), \\
& \gamma_{3}=(1,1, c, \gamma(4), \ldots, \gamma(n)), \\
& \delta_{0}=(0, c, 0, \delta(4), \ldots, \delta(n)), \\
& \delta_{1}=(1, c, 0, \delta(4), \ldots, \delta(n)), \\
& \delta_{2}=(0, c, 1, \delta(4), \ldots, \delta(n)), \\
& \delta_{3}=(1, c, 1, \delta(4), \ldots, \delta(n)),
\end{aligned}
$$

Пусть $\phi_{1}\left(\gamma_{0}\right)=\phi_{1}\left(\delta_{0}\right)$. Положим

$$
\alpha_{1}=\gamma_{2-2 c}, \alpha_{2}=\delta_{3-2 c}, \alpha_{3}=\gamma_{3 c}, \beta_{1}=\gamma_{2-2 c}, \beta_{2}=\gamma_{3-2 c}, \beta_{3}=\delta_{2 c} .
$$

Тогда верно неравенство

$$
\phi_{1}\left(v\left(\alpha_{1}, \alpha_{2}, \alpha_{3}\right)\right)=\phi_{1}\left(\gamma_{1+2 c}\right) \neq \phi_{1}\left(\gamma_{2-2 c}\right)=\phi_{1}\left(v\left(\beta_{1}, \beta_{2}, \beta_{3}\right)\right) .
$$


Аналогично рассмотрим случай $\phi_{1}\left(\gamma_{0}\right) \neq \phi_{1}\left(\delta_{0}\right)$. В этом случае положим $\alpha_{1}=$ $=\gamma_{1+2 c}, \alpha_{2}=\delta_{2-2 c}, \alpha_{3}=\delta_{3 c}$ и при $\mathrm{c}=0$ положим $\beta_{1}=\gamma_{0}, \beta_{2}=\gamma_{1}, \beta_{3}=\gamma_{2}$, a при $c=1-\beta_{1}=\delta_{0}: \beta_{2}=\delta_{1}, \beta_{3}=\gamma_{0}$. Легко показать, что в обоих случаях

$$
\phi_{1}\left(v\left(\alpha_{1}, \alpha_{2}, \alpha_{3}\right)\right) \neq \phi_{1}\left(v\left(\beta_{1}, \beta_{2}, \beta_{3}\right)\right) .
$$

Пусть теперь $c=\gamma(3) \neq \delta(2)=c \oplus 1$. Тогда, перегруппировав в (7), а затем и в (11) векторы $\delta_{i}, i=0, \ldots, 3$, в порядке $\delta_{2}, \delta_{3}, \delta_{0}, \delta_{1}$ и соответствующим образом их переобозначив, дословно повторяем проведенные ранее рассуждения.

Во всех возможных случаях построены наборы векторов $\left\{\alpha_{1}, \alpha_{2}, \alpha_{3}\right\}$, $\left\{\beta_{1}, \beta_{2}, \beta_{3}\right\}$, для которых выполнены условия (9) и (10). Полученные противоречия показывают, что функция $\phi_{1}$ не может существенно зависеть более чем от одной переменной.

2. Случай $\phi\left(V_{n}\right)=V_{n}$. Тогда $\phi-$ биекция, т.е. подстановка на множестве $V_{n}$. Построим следующий гомоморфизм. Положим

$$
\hat{\phi}_{i}(x)=\left(0, \ldots, 0, \phi_{i}(x), 0, \ldots, 0\right): V_{n} \rightarrow V_{n}, \quad i=1,2, \ldots, n .
$$

Тогда очевидно, что $\hat{\phi}_{i}-$ эндоморфизм $\Omega_{n}$, причем $\hat{\phi}_{i}\left(V_{n}\right) \neq V_{n}$. К такому гомоморфизму можно применить рассуждения шага 1 и получить, что функция $\phi_{i}$ существенно зависит не более чем от одной переменной. Так как эти рассуждения можно провести для любого $i \in\{1,2, \ldots, n\}$, то все координатные функции эндоморфизма $\phi$ существенно зависят не более, чем от одной переменной, что и требовалось доказать.

Первая часть теоремы доказана в прямую сторону. Докажем ее в обратную сторону.

3. Пусть $\phi=\left(\phi_{1}, \ldots, \phi_{n}\right): V_{n} \rightarrow V_{n}$ и каждая координатная функция $\phi_{1}, \ldots, \phi_{n}$ существенно зависит не более чем от одной переменной. Докажем, что в этом случае $\phi-$ эндоморфизм алгебры $\Omega_{n}$. Пусть

$$
\alpha_{1}, \alpha_{2}, \alpha_{3} \in V_{n}, \gamma_{1}=\phi\left(\alpha_{1}\right), \gamma_{2}=\phi\left(\alpha_{2}\right), \gamma_{3}=\phi\left(\alpha_{3}\right) .
$$

Покажем, что

$$
\phi\left(v\left(\alpha_{1}, \alpha_{2}, \alpha_{3}\right)\right)=v\left(\gamma_{1}, \gamma_{2}, \gamma_{3}\right) .
$$

Ввиду того что функция $v$ действует покоординатно, равенство (12) эквивалентно условию

$$
\phi_{j}\left(v\left(\alpha_{1}, \alpha_{2}, \alpha_{3}\right)\right)=v\left(\gamma_{1}(j), \gamma_{2}(j), \gamma_{3}(j)\right)
$$


для всякого $j \in\{1,2, \ldots, n\}$. Поэтому достаточно доказать равенство (13) для одной координаты, например, первой. Пусть $\phi_{1}\left(x_{1}, \ldots, x_{n}\right)=a_{1} x_{i} \oplus b_{1}$. Тогда

$$
\gamma_{1}(1)=a_{1} \alpha_{1}(i) \oplus b_{1}, \quad \gamma_{2}(1)=a_{1} \alpha_{2}(i) \oplus b_{1}, \quad \gamma_{3}(1)=a_{1} \alpha_{3}(i) \oplus b_{1} .
$$

Если $a_{1}=0$, то $\gamma_{1}(1)=\gamma_{2}(1)=\gamma_{3}(1)=b_{1}$. Но в этом случае

$$
\phi\left(v\left(\alpha_{1}, \alpha_{2}, \alpha_{3}\right)\right)(1)=b_{1}=v\left(\gamma_{1}(1), \gamma_{2}(1), \gamma_{3}(1)\right),
$$

т. е. равенство (13) выполняется по первой координате. Пусть теперь $a_{1}=1$. Тогда $\gamma_{1}(1)=\alpha_{1}(i) \oplus b_{1}, \gamma_{2}(1)=\alpha_{2}(i) \oplus b_{1}, \gamma_{3}(1)=\alpha_{3}(i) \oplus b_{1}$. При $b_{1}=0$ равенство (13) также выполняется очевидным образом. Пусть $b_{1}=1$, т. е. $\gamma_{1}(1)=\alpha_{1}(i) \oplus 1, \gamma_{2}(1)=\alpha_{2}(i) \oplus 1, \gamma_{3}(1)=\alpha_{3}(i) \oplus 1$. Тогда в силу самодвойственности функции $v$ получаем

$$
\begin{aligned}
& v\left(\gamma_{1}(1), \gamma_{2}(1), \gamma_{3}(1)\right)=v\left(\alpha_{1}(i) \oplus 1, \alpha_{2}(i) \oplus 1, \alpha_{3}(i) \oplus 1\right)= \\
& =v\left(\alpha_{1}(i), \alpha_{2}(i), \alpha_{3}(i)\right) \oplus 1=\phi_{1}\left(v\left(\alpha_{1}(i), \alpha_{2}(i), \alpha_{3}(i)\right)\right) .
\end{aligned}
$$

Из проведенных рассуждений следует, что $\phi-$ эндоморфизм алгебры $\Omega_{n}$. Первая часть теоремы полностью доказана.

Перейдем к доказательству второй части теоремы.

4. Пусть $\phi=\left(\phi_{1}, \ldots, \phi_{n}\right)$. Ввиду доказанного в пунктах 1-3 будем считать, что

$$
\phi_{i}\left(x_{1}, \ldots, x_{n}\right)=a_{i} x_{r_{i}} \oplus b_{i}, i=1,2, \ldots, n .
$$

Ясно, что путем подходящей перестановки переменных преобразование $\phi=$ $=\left(\phi_{1}, \ldots, \phi_{n}\right)$ можно привести к виду

$$
\phi(x)=x A \oplus \beta
$$

с матрицей

$$
A=\left(\begin{array}{ccc}
P & P^{\prime} & 0 \\
0 & 0 & 0
\end{array}\right)
$$

где $P$ - подстановочная $m \times m$-матрица для некоторого $m \leq n, P^{\prime}-$ $m \times k$-матрица для некоторого $k \leq n-m$, содержащая ровно одну единицу в каждом столбце. При этом $m+k$ - число ненулевых коэффициентов $a_{i}$ в правой части выражения для $\phi_{i}$. Определим матрицу размера $n \times m$ вида $A^{\prime}=\left(\begin{array}{c}P^{-1} \\ 0\end{array}\right)$. Пусть $\pi-$ подстановка, соответствующая матрице $P$. Построим отображение $\theta: \phi\left(V_{n}\right) \rightarrow V_{m}$ следующим образом:

$$
\theta(x)=x A^{\prime} \oplus \beta^{\prime}
$$


где $\beta^{\prime} \in V_{m}$ и $\beta^{\prime}(i)=\beta\left(\pi^{-1}(i)\right), \quad i=1,2, \ldots, m$. Поскольку данное отображение является аффинным отображением с матрицей ранга $m$, множество $\phi\left(V_{n}\right)$ - аффинное многообразие пространства $V_{n}$, то очевидно, что $\theta-$ биекция. Покажем, что это отображение является гомоморфизмом алгебр, т. е. для любых $\gamma_{1}, \gamma_{2}, \gamma_{3} \in \phi\left(V_{n}\right)$ выполняется свойство

$$
\theta\left(v\left(\gamma_{1}, \gamma_{2}, \gamma_{3}\right)\right)=v\left(\theta\left(\gamma_{1}\right), \theta\left(\gamma_{2}\right), \theta\left(\gamma_{3}\right)\right)
$$

Как и в п. 3 настоящего доказательства, покажем, что для всякого $i \in$ $\in\{1,2, \ldots, m\}$

$$
\theta\left(v\left(y_{1}, y_{2}, y_{3}\right)\right)(i)=v\left(\theta\left(y_{1}\right)(i), \theta\left(y_{2}\right)(i), \theta\left(y_{3}\right)(i)\right)
$$

Если $\gamma_{1}, \gamma_{2}, \gamma_{3} \in \phi\left(V_{n}\right)$, то для некоторых $\alpha_{1}, \alpha_{2}, \alpha_{3} \in V_{n}$ выполнены равенства

$$
\gamma_{j}(i)=\alpha_{j}(\pi(i)) \oplus b(i), i=1, \ldots, m, j=1,2,3 .
$$

Тогда для $i=1,2, \ldots, m$ верна цепочка равенств

$$
\begin{aligned}
& v\left(\theta\left(\gamma_{1}\right)(i), \theta\left(\gamma_{2}\right)(i), \theta\left(\gamma_{3}\right)(i)\right)= \\
& =v\left(\gamma_{1}\left(\pi^{-1}(i)\right) \oplus b\left(\pi^{-1}(i)\right), \gamma_{2}\left(\pi^{-1}(i)\right) \oplus b\left(\pi^{-1}(i)\right), \gamma_{3}\left(\pi^{-1}(i)\right) \oplus b\left(\pi^{-1}(i)\right)\right)= \\
& =v\left(\gamma_{1}\left(\pi^{-1}(i)\right), \gamma_{2}\left(\pi^{-1}(i)\right), \gamma_{2}\left(\pi^{-1}(i)\right)\right) \oplus b\left(\pi^{-1}(i)\right)= \\
& =v\left(\gamma_{1}, \gamma_{2}, \gamma_{2}\right)\left(\pi^{-1}(i)\right) \oplus b\left(\pi^{-1}(i)\right)=\theta\left(v\left(\gamma_{1}(i), \gamma_{2}(i), \gamma_{3}(i)\right)\right) .
\end{aligned}
$$

Таким образом, показано, что $\theta$ - гомоморфизм и, следовательно, изоморфизм.

5. Докажем последнюю часть теоремы: совпадение множества гомоморфных образов $\phi\left(V_{n}\right)$ с множествами выполняющих векторов мультиаффинных биюнктивных функций. Рассмотрим множество $\phi\left(V_{n}\right)$. В самом деле, $y=(y(1), \ldots, y(n)) \in \phi\left(V_{n}\right)$ тогда и только тогда, когда для некоторого вектора $(x(1), \ldots, x(n)) \in V_{n}$ выполняется система равенств

$$
\left\{\begin{array}{l}
y(1)=a_{1} x\left(r_{1}\right) \oplus b_{1}, \\
\cdots \quad \cdots \quad \cdots \quad \cdots \\
y(n)=a_{n} x\left(r_{n}\right) \oplus b_{n}
\end{array}\right.
$$

Очевидно, что если $y_{1}, y_{2}, y_{3} \in \phi\left(V_{n}\right)$, т. е. удовлетворяют системе (14), то и вектор $z=y_{1} \oplus y_{2} \oplus y_{3}$ также удовлетворяет системе (14). Поэтому, положив $f=f_{\phi}\left(V_{n}\right)$, получаем, что $f-$ мультиаффинна по критерию мультиаффинности (см. [3]). Теперь рассмотрим вектор $u=v\left(y_{1}, y_{2}, y_{3}\right)$. Пусть 
$y_{1}=\phi\left(x_{1}\right), y_{2}=\phi\left(x_{2}\right), y_{3}=\phi\left(x_{3}\right)$. Тогда для произвольной координаты $i \in\{1,2, \ldots, n\}$ выполнено равенство

$$
\begin{aligned}
& u(i)=y_{1}(i) y_{2}(i) \oplus y_{2}(i) y_{3}(i) \oplus y_{1}(i) y_{3}(i)= \\
& =\left(a_{i} x_{1}\left(r_{i}\right) \oplus b_{i}\right)\left(a_{i} x_{2}\left(r_{i}\right) \oplus b_{i}\right) \oplus\left(a_{i} x_{2}\left(r_{i}\right) \oplus b_{i}\right)\left(a_{i} x_{3}\left(r_{i}\right) \oplus b_{i}\right) \oplus \\
& \oplus\left(a_{i} x_{1}\left(r_{i}\right) \oplus b_{i}\right)\left(a_{i} x_{3}\left(r_{i}\right) \oplus b_{i}\right)= \\
& =a_{i}\left(x_{1}\left(r_{i}\right) x_{2}\left(r_{i}\right) \oplus x_{2}\left(r_{i}\right) x_{3}\left(r_{i}\right) \oplus x_{1}\left(r_{i}\right) x_{3}\left(r_{i}\right)\right) \oplus b_{i}= \\
& =a_{i} v\left(y_{1}(i), y_{2}(i), y_{3}(i)\right) \oplus b_{i},
\end{aligned}
$$

т. е. $u=v\left(\phi\left(x_{1}\right), \phi\left(x_{2}\right), \phi\left(x_{3}\right)\right)$ и $u \in E_{f}$. Значит, по критерию биюнктивности, $f$ - биюнктивна, и $f \in A \cap B i$.

Обратно, пусть $f \in A \cap B i$. Покажем, что найдется такой эндоморфизм $\phi$, что $\phi\left(V_{n}\right)=E_{f}$. В силу доказанных выше утверждений достаточно найти такое отображение $\phi \in \Xi_{n}$, что $\phi\left(V_{n}\right)=E_{f}$.

Пусть $f\left(x_{1}, \ldots, x_{n}\right)=\bigwedge_{i=1}^{k} x_{s_{i}}^{a_{i}} \bigwedge_{j=1}^{t}\left(x_{p_{j, 1}} \oplus x_{p_{j, 2}} \oplus b_{j}\right)-$ мультиаффинная биюнктивная функция. Определим преобразование $\phi$, положив

$$
\begin{aligned}
& \phi_{s_{i}}=a_{i}, i=1, \ldots, k, \\
& \phi_{p_{j, 1}}=x_{p_{j, 1},}, \phi_{p_{j, 2}}=x_{p_{j, 1}} \oplus b_{j}, j=1,2, \ldots, t \\
& \phi_{k}=x_{k}, k \in\{1,2, \ldots, n\} \backslash\left(\left\{s_{1}, \ldots, s_{k}\right\} \cup\left\{p_{1,1}, \ldots, p_{t, 1}\right\} \cup\left\{p_{1,2}, \ldots, p_{t, 2}\right\}\right) .
\end{aligned}
$$

Легко видеть, что всякий вектор из $\phi\left(V_{n}\right)$ является выполняющим для $f$, т. е. $\phi\left(V_{n}\right) \subset E_{f}$. С другой стороны, пусть $\gamma=\left(c_{1}, \ldots, c_{n}\right) \in E_{f}$. Тогда рассмотрим такой вектор $\beta=\left(b_{1}, \ldots, b_{n}\right)$, что

$$
\begin{aligned}
& b_{p_{j, 1}}=c_{p_{j, 1}}, \quad j=1,2, \ldots, t, \\
& b_{k}=c_{k}, k \in\{1,2, \ldots, n\} \backslash\left(\left\{s_{1}, \ldots, s_{k}\right\} \cup\left\{p_{1,1}, \ldots, p_{t, 1}\right\} \cup\left\{p_{1,2}, \ldots, p_{t, 2}\right\}\right) .
\end{aligned}
$$

Очевидно, что $\phi(\beta)=\gamma$ и, следовательно, $\phi\left(V_{n}\right)=E_{f}$. Теорема полностью доказана.

Следует отметить, что для числа гомоморфных образов алгебры $\Omega_{n}$ можно использовать как точные формулы С. П. Горшкова ( [4]), так и асимптотику В.Н. Сачкова ( [8]). Для алгебр $\Omega_{n}^{r}, r \geq 3$, эндоморфизмы описывает следующая теорема.

Теорема 6. Пусть $\phi=\left(\phi_{1}, \ldots, \phi_{n}\right): V_{n} \rightarrow V_{n}$. Для того чтобы выполнялось условие $\phi \in \operatorname{End}\left(\Omega_{n}^{r}\right)$, необходимо и достаточно, чтобы $\phi \in \Xi_{n}^{0}$. Получаемые при таких эндоморфизмах образы алгебры $\Omega_{n}^{r}$ являются подалгебрами $\Omega_{n}^{r}$, изоморфными алгебрам $\Omega_{0}^{r}, \ldots, \Omega_{n-1}^{r}, \Omega_{n}^{r}$. 
Доказательство. Очевидно, что End $\left(\Omega_{n}^{r}\right) \supset \Xi_{n}^{0}$. Докажем прямое включение. Пусть $\phi=\left(\phi_{1}, \ldots, \phi_{n}\right) \in \operatorname{End}\left(\Omega_{n}^{r}\right)$. Проведем рассуждения первой части теоремы 5 вплоть до получения формул (8). Далее, на основании формул (7) и (8) существуют такие наборы векторов $\left\{\alpha_{1}, \ldots, \alpha_{r+1}\right\},\left\{\beta_{1}, \ldots, \beta_{r+1}\right\}, \quad$ что $\quad \alpha_{1} \varepsilon_{\phi_{1}} \beta_{1}, \ldots, \alpha_{r+1} \varepsilon_{\phi_{1}} \beta_{r+1}$, но $\phi_{1}\left(v_{r}\left(\alpha_{1}, \ldots, \alpha_{r+1}\right)\right) \neq \phi_{1}\left(v_{r}\left(\beta_{1}, \ldots, \beta_{r+1}\right)\right)$. Указанные наборы строятся аналогично тому, как это делалось в доказательстве теоремы 5 , с учетом равенств

$$
\alpha_{3}=\ldots=\alpha_{r+1}, \quad \beta_{3}=\ldots=\beta_{r+1} .
$$

Полученные противоречия показывают, что функция $\phi_{1}$ не может существенно зависеть более чем от одной переменной и должна лежать в множестве $\Xi_{n}$.

Пусть функция $\phi_{1}$ существенно зависит в точности от одной переменной. Покажем, что для этой функции выполняется равенство: $\phi_{1}\left(0_{n}\right)=0$. Предположим противное: $\phi_{1}\left(0_{n}\right)=1$. Без ограничения общности считаем, что $\phi_{1}\left(x_{1}, \ldots, x_{n}\right)=x_{1} \oplus 1$. Рассмотрим векторы $\alpha_{1}=\ldots=\alpha_{r-1}=(10 \ldots 0)$, $\alpha_{r}=\alpha_{r+1}=(0 \ldots 0)$. В этом случае $v_{r}\left(\alpha_{1}, \ldots, \alpha_{r+1}\right)=\alpha_{1}=(10 \ldots 0)$.

При этом $\phi_{1}\left(v_{r}\left(\alpha_{1}, \ldots, \alpha_{r+1}\right)\right)=\phi_{1}\left(\alpha_{1}\right)=0$. Однако, первая координата вектора $v_{r}\left(\phi\left(\alpha_{1}\right), \ldots, \phi\left(\alpha_{r+1}\right)\right)$, очевидно, равна 0. Поэтому $\phi\left(v_{r}\left(\alpha_{1}, \ldots, \alpha_{r+1}\right)\right) \neq v_{r}\left(\phi\left(\alpha_{1}\right), \ldots, \phi\left(\alpha_{r+1}\right)\right)$. Поэтому исходное предположение неверно, и $\phi \in \Xi_{n}^{0}$.

2. Оставшаяся часть теоремы доказывается аналогично пунктам 2-4 доказательства теоремы 5 .

\section{4. Свойства систем образующих подалгебр алгебры $\Omega_{n}$}

Обратимся теперь к некоторым свойствам систем образующих подалгебр алгебры $\Omega_{n}$ (случай $r=2$ ) и исследуем зависимость между свойствами графов 2-КНФ биюнктивных функций и системами образующих соответствующих алгебр.

В работе [3] показано, что множество выполняющих векторов слабо положительной функции $f\left(x_{1}, \ldots, x_{n}\right)$ вида (3) образует нижнюю полурешетку, определяемую множеством неразложимых векторов $R_{f}$, где под неразложимыми векторами понимаются выполняющие векторы функции $f$, которые не представимы в виде покоординатной дизъюнкции других выполняющих векторов. 
Теорема 7. Пусть $f \in W P$ не имеет имплицент вида $x_{i}^{a}, 1 \leq i \leq n$, $a \in\{0,1\}, R_{f} \subset V_{n}-$ множество неразложимых векторов функции $f$. Тогда верно включение $E_{f} \subset\left\langle R_{f} \cup\left\{1_{n}\right\}\right\rangle$, обращзающееся в равенство при $f \in W P \cap$ $\cap$ Вi. Если же $f \in W P \cap B i$ u $1_{n} \in R_{f}$, то для некоторого $i \in 1, \ldots, n$ выполняется условие

$$
f\left(x_{1}, \ldots, x_{n}\right) \leq \bigwedge_{j \neq i}\left(\bar{x}_{i} \vee x_{j}\right)
$$

Доказательство. Если $f \in W P$, то в соответствии с критерием слабой положительности (см. [3]) множество $E_{f}$ замкнуто относительно покоординатной дизъюнкции, и, если $R_{f}=\left\{\alpha_{1}, \ldots, \alpha_{t}\right\}$, каждый ненулевой вектор $\beta \in E_{f}$ может быть представлен в виде $\beta=\alpha_{i_{1}} \vee \ldots \vee \alpha_{i_{s}}$ для некоторых $s \in\{1,2, \ldots, t\}, \quad 1 \leq i_{1}<\ldots<i_{s} \leq t$. Кроме того, из условия теоремы следует, что $1_{n} \in E_{f}$. Поскольку $v\left(\alpha, \beta, 1_{n}\right)=\alpha \vee \beta$ для любых $\alpha, \beta \in V_{n}$, очевидно, что $E_{f} \subset\left\langle R_{f} \cup\left\{1_{n}\right\}\right\rangle$.

Если же $f \in W P \cap B i$, то, поскольку $R_{f} \subset E_{f}$, верна цепочка включений $\left\langle R_{f}\right\rangle \subset E_{f} \subset\left\langle R_{f} \cup\left\{1_{n}\right\}\right\rangle$, и так как $1_{n} \in E_{f}$, то $E_{f}=\left\langle R_{f} \cup\left\{1_{n}\right\}\right\rangle$.

Докажем последнее утверждение теоремы. Пусть $R_{f}=\left\{\alpha_{1}, \ldots, \alpha_{t}\right\}$, $\alpha_{t}=1_{n}$. Рассмотрим вектор $\beta=\alpha_{1} \vee \ldots \vee \alpha_{t-1}$. Если $\beta=1_{n}$, то получаем противоречие с условием неразложимости вектора $1_{n}$.Следовательно, для некоторого $i \in\{1, \ldots, n\} \beta(i)=0$, откуда $\alpha_{1}(i)=\ldots=\alpha_{t}(i)=0$. Это означает, что единственным вектором множества $E_{f}$, имеющим единицу на $i$-й координате, является вектор $1_{n}$, откуда и следует последнее утверждение теоремы.

Пусть биюнктивная функция $f\left(x_{1}, \ldots, x_{n}\right)$ задана своей 2-КНФ

$$
f\left(x_{1}, \ldots, x_{n}\right)=\stackrel{t}{i}_{i=1}^{t}\left(x_{s_{i 1}}^{a_{i 1}} \vee x_{s_{i 2}}^{a_{i 2}}\right) .
$$

Определим множества $X=\left\{x_{1}, \ldots, x_{n}\right\}, \bar{X}=\left\{\bar{x}_{1}, \ldots, \bar{x}_{n}\right\}$. Для 2-КНФ вида (16) определим ориентированный граф $G=(V, \varepsilon)$ :

$$
V=X \cup \bar{X}, \quad \varepsilon=\left\{\left(x_{s_{i 1}}^{a_{i 1} \oplus 1}, x_{s_{i 2}}^{a_{i 2}}\right),\left(x_{s_{i 2}}^{a_{i 2} \oplus 1}, x_{s_{i 1}}^{a_{i 1}}\right), i=1, \ldots, t\right\} .
$$

Известен следующий результат, полученный Тарьяном. Одно из его доказательств приведено в [6].

Теорема 8 ([6]). Элементарная дизъюнкция $x_{i}^{a} \vee x_{j}^{b}$ является простой имплицентой функичи $f$, заданной 2-КНФ (2), тогда и только тогда, когда в графе $G$, заданном (17), существуют пути из вершины $x_{i}^{a \oplus 1}$ в вершину $x_{j}^{b}$ и из вершины $x_{j}^{b \oplus 1}$ в вершину $x_{i}^{a}$. 
Напомним, что класс $W P \cap W N$ является подклассом класса $B i$, содержащим функции вида

$$
f \equiv\left(\underset{i=1}{t_{1}} x_{s_{i}}^{a_{i}}\right) \wedge\left(\underset{i=t_{1}+1}{\stackrel{t}{\&}}\left(\bar{x}_{s_{i 1}}^{\vee} x_{s_{i 2}}^{)}\right)\right.
$$

При этом множество выполняющих векторов $E_{f}$ такой функции образует дистрибутивную решетку. Для такой решетки можно сравнительно легко описать как множества неразложимых векторов $R_{f}$, так и множества конеразложимых векторов $c o-R_{f}$. Наиболее наглядно эти множества описываются, если функция $f$ не имеет аффинных имплицент. В этом случае ее можно записать в виде

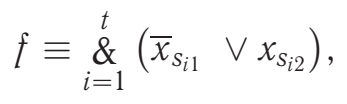

причем все имплиценты в правой части (17) простые.

В графе 2-КНФ такой функции нет дуг, соединяющих вершины множеств $X$ и $\bar{X}$, и можно рассматривать его подграф $G_{1}$, порожденный множеством вершин $X: G_{1}=G(f)(X)$. Множество дуг такого графа обозначим через $\varepsilon_{1}$. В дальнейшем считаем, что данный граф является графом минимальной 2-КНФ. В соответствии с результатами статьи [6], отсутствие аффинных имплицент функции $f\left(x_{1}, \ldots, x_{n}\right)$ гарантирует отсутствие контуров в графе $G_{1}$ и, соответственно, сильно связных компонент. Минимальность КНФ (17) означает, в свою очередь, что в графе $G_{1}$ нет дуг, соединяющих начала и концы путей длины не меньше 2 .

Лемма 2. Пусть $f \in W P \cap W N$ и граф $G_{1}$ не имеет сильно связных компонент. Тогда множество $R_{f}$ состоит из $n+1$ векторов $R_{f}=$ $=\left\{\alpha_{0}, \alpha_{1}, \ldots, \alpha_{n}\right\}$, где $\alpha_{0}=0_{n}$, а векторы $\alpha_{i}, i=1, \ldots, n$, построены по принципу $\alpha_{i}(i)=1$ и $\alpha_{i}(j)=1$ для всех таких $j$, что вершина $x_{j}$ достижима из вершины $x_{i}$, т.е. в графе $G_{1}$ существует ориентированный путь из вериины $x_{i}$ в вериину $x_{j}$.

Доказательство. Воспользуемся результатами из статьи [3]. Поскольку $W P \cap W N \subset W P$, множество $E_{f}$, являющееся решеткой, является, в частности, нижней полурешеткой. Для нахождения множества $R_{f}$ воспользуемся описанным в указанной работе подходом. В соответствии с результатом [3] верно равенство

$$
R_{f} \backslash\left\{0_{n}\right\}=\bigcup_{i=1}^{n} \phi_{i}^{-1}\left(W_{f_{i}^{1}}\right),
$$


где $W_{f_{i}^{1}}-$ множество нижних единиц подфункции $f_{i}^{1}$ функции $f$, a

$$
\phi_{i}^{-1}(W)=\left\{\left(a_{1}, \ldots, a_{i-1}, 1, a_{i}, \ldots, a_{n-1}\right):\left(a_{1}, \ldots, a_{n-1}\right) \in W \subset V_{n-1}\right\} .
$$

Опишем множество $W_{f_{i}^{1}}$ для любого $i \in\{1,2, \ldots, n\}$. Пусть, без ограничения общности, $i=1$. Перепишем (18) в следующем виде:

$$
f \equiv \stackrel{k}{i=1}_{i}^{k}\left(\bar{x}_{1} \vee x_{s_{i}}\right) \underset{i=k+1}{\stackrel{m}{\&}}\left(\bar{x}_{s_{i}} \vee x_{1}\right) h\left(x_{2}, \ldots, x_{n}\right)
$$

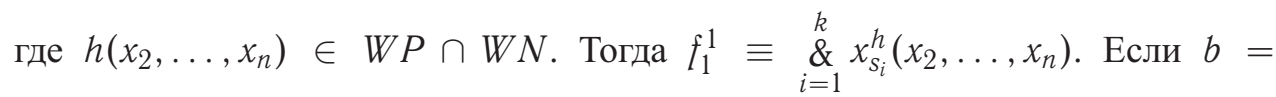
$=\left(b_{2}, \ldots, b_{n}\right) \in E_{f_{1}^{1}}$, то

$$
b_{s_{1}}=\ldots=b_{s_{k}}=1 \text {. }
$$

Поскольку $h(0, \ldots, 0)=1$, для построения нижних единиц необходимо описать все векторы из $E_{h}$, удовлетворяющие условию (20).

Из теоремы 8 следует, что если $\beta=\left(b_{2}, \ldots, b_{n}\right) \in E_{f_{1}^{1}}$ и выполнено (20), то для всякого такого $j$, что вершина $x_{j}$ достижима из одной из вершин $x_{s_{1}}, \ldots, x_{s_{k}}$, должно выполняться условие $b_{j}=1$. Если же вершина $x_{j}$ достижима хотя бы из одной из вершин $x_{s_{1}}, \ldots, x_{s_{k}}$, но $b_{j}=0$, то вектор $\beta=\left(b_{2}, \ldots, b_{n}\right) \notin E_{f_{1}^{1}}$. Следовательно, определенный выше вектор $\beta$ будет единственной нижней единицей $f_{i}^{1}$, а вектор $\phi_{1}^{-1}(\beta)=\left(1, b_{2}, \ldots, b_{n}\right)-$ неразложимым вектором решетки $E_{f}$.

Утверждение 5. Пусть $f \in W P \cap W N$ u граф $G(f)(X)$ не имеет сильно связных компонент. Положим $g\left(x_{1}, \ldots, x_{n}\right)=f\left(x_{1} \oplus 1, \ldots, x_{n} \oplus 1\right) \in$ $\in W P \cap W N$. Тогда множества неразложимых и конеразложимых векторов функичий f $и$ g связаны соотношениями

$$
R_{f}=\left(c o-R_{g}\right) \oplus 1_{n}, \quad c o-R_{f}=R_{g} \oplus 1_{n}
$$

Доказательство. Заметим, что $E_{g}=E_{f} \oplus 1_{n}$. Далее, если $\alpha_{i_{1}}, \ldots, \alpha_{i_{k}} \in$ $\in R_{f}$ для $i_{1}, \ldots, i_{k} \in\{1, \ldots, n\}$ и $\alpha_{i_{1}} \vee \ldots \vee \alpha_{i_{k}}=\beta \in E_{f}$, то $\left(\alpha_{i_{1}} \oplus 1_{n}\right) \wedge$ $\ldots \wedge\left(\alpha_{i_{k}} \oplus 1_{n}\right)=\beta \oplus 1_{n} \in E_{g}$, и наоборот.

В силу того что граф $G_{1}$ функции вида (19) не имеет сильно связных компонент, для каждой его вершины $x_{i}, i=1, \ldots, n$, можно рассмотреть подграф $T\left(x_{i}\right)$, построенный на вершинах, достижимых из $x_{i}$. Данный граф, очевидно, имеет единственную начальную вершину $x_{i}$ и не имеет контуров. 
Теорема 9. Пусть выполнены условия леммы 2 и для некоторой вершины $x_{i}, i=1,2, \ldots, n$, графа $G_{1}$ полустепень захода $\operatorname{deg}^{+}\left(x_{i}\right)$ удовлетворяет условию $\operatorname{deg}^{+}\left(x_{i}\right) \geq 2$. Тогда если в графе существуют такие вериины $x_{j}, x_{k}$, что $\left(x_{j}, x_{i}\right),\left(x_{k}, x_{i}\right) \in \epsilon_{1}$, и при этом выполнено условие $T\left(x_{j}\right) \cap T\left(x_{k}\right)=T\left(x_{i}\right)$, $m o\left\langle R_{f}\right\rangle=\left\langle R_{f} \backslash\left\{\alpha_{i}\right\}\right\rangle$.

Доказательство. Достаточно доказать, что $\alpha_{i}=v\left(\alpha_{j}, \alpha_{k}, 0_{n}\right)$. Без ограничения общности положим $j=1, k=2, i=3$. Тогда

$$
\alpha_{1}=(1,0,1, \ldots), \alpha_{2}=(0,1,1, \ldots), \alpha_{3}=(0,0,1, \ldots) .
$$

При этом все вершины, достижимые из $\alpha_{3}$, будут достижимыми из вершин $\alpha_{1}, \alpha_{2}$. Это означает, что если $\alpha_{3}(m)=1$, то $\alpha_{1}(m)=\alpha_{2}(m)=1, m=$ $1,2, \ldots, n$. Пусть $\alpha_{3}(m)=0$ для некоторого $m, 4 \leq m \leq n$. Так как $T\left(x_{j}\right) \cap$ $\cap T\left(x_{k}\right)=T\left(x_{i}\right)$, то выполняется свойство $\alpha_{1}(m) \& \alpha_{2}(m)=0$. Из проведенных рассуждений вытекает, что $\alpha_{3}=v\left(\alpha_{1}, \alpha_{2}, 0_{n}\right)$, откуда следует утверждение теоремы.

Доказанная теорема дает возможность дальнейших исследований по построению минимальных систем образующих подалгебр алгебры $\Omega_{n}$.

\section{Список литературы}

1. Schaefer T. Complexity of satisfiability problems. - Proc. $10^{\text {th }}$ Annu. ACM Symp. theory of computing machinery, 1978, p. 216-226.

2. Горшков С. П. Применение теории NР-полных задач для оценки сложности решения систем булевых уравнений. - Обозрение прикл. и промышл. матем., 1995, т. 2, вып. 3, с. 325-398.

3. Горшков С.П. О сложности распознавания мультиаффинности, биюнктивности, слабой положительности и слабой отрицательности булевых функций. - Обозрение прикл. и промышл. матем., 1997, т. 4, вып. 2 , c. 216-237.

4. Горшков С.П. О пересечениях классов мультиаффинных, биюнктивных, слабо положительных и слабо отрицательных булевых функций. Обозрение прикл. и промышл. матем., 1997, т. 4, вып. 2, с. 238-259.

5. Горшков С.П., Тарасов А.В. Теоретико-сложностной подход к оценке сложности решения систем булевых уравнений. - Матер. 4-й Междунар. научн. конф. по пробл. безопасн. и противодействия терроризму (МаБИТ-2008), МГУ, 2008, т. 2, с. 36-45. 
6. Тарасов A.B. О свойствах функций, представимых в виде 2-КНФ. Дискретная математика, 2001, т. 13, вып. 4, с.99-115.

7. Кон П. Универсальная алгебра. - М.: Мир, 1968.

8. Горшков С.П., Тарасов А.В. О максимальных группах инвариантных преобразований мультиаффинных, биюнктивных, слабо положительных и слабо отрицательных булевых функций. - Дискретная математика, 2009, т. 21, вып. 2, с. 94-101.

9. Сачков B.H. Разбиения с поглощениями и противоречивые разбиения множеств. - Труды по дискретной математике, т. 4, М.: ФИЗМАТЛИТ, 2001, c. 201-222.

10. Сачков В.Н. Введение в комбинаторные методы дискретной математики. - М.: Наука, 1982. 